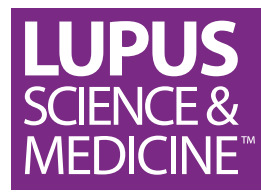

\title{
Repository corticotropin injection in patients with persistently active SLE requiring corticosteroids: post hoc analysis of results from a two-part, 52- week pilot study
}

\author{
Richard A Furie, ${ }^{1}$ Margaret Mitrane, ${ }^{2}$ Enxu Zhao, ${ }^{3}$ Patrice M Becker ${ }^{3}$
}

To cite: Furie RA, Mitrane M, Zhao E, et al. Repository corticotropin injection in patients with persistently active SLE requiring corticosteroids: post hoc analysis of results from a two-part, 52-week pilot study. Lupus Science \& Medicine 2017;4:e000240. doi:10.1136/ lupus-2017-000240

Received 15 August 2017 Revised 29 September 2017 Accepted 11 November 2017

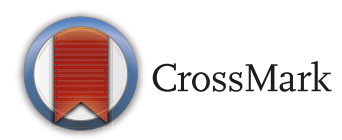

${ }^{1}$ Hofstra Northwell School of Medicine, Northwell Health, Great Neck, New York, USA ${ }^{2}$ Manhattan BioPharm Consultants LLC, New York, New York, USA

${ }^{3}$ Mallinckrodt ARD Inc., Bedminster, New Jersey, USA

Correspondence to Dr Richard A Furie; RFurie@ northwell.edu

\begin{abstract}
Objective Post hoc analyses evaluated the effectiveness and safety of repository corticotropin injection (RCI) in patients with persistently active SLE over 52 weeks. Methods Patients were initially randomised to $40 \mathrm{U}$ daily or $80 \mathrm{U}$ every other day $\mathrm{RCI}(\mathrm{n}=26)$ or placebo $(n=12)$ for the 8-week double-blind period. Completers entered the open-label extension (OLE; $n=33$ ) receiving 16, 40 or $80 \mathrm{U} \mathrm{RCl} 1-3$ times/week and were followed through week 52. Outcomes included proportion of responders based on a novel index (resolution of joint or skin activity using hybrid Systemic Lupus Erythematosus Disease Activity Index (hSLEDAl) without any worsening British Isles Lupus Assessment Group (BILAG) scores in other organ systems) or revised novel index (using SLE Responder Index (SRI) definition of BILAG worsening (1A or $2 B)$ ), proportion of responders by SRI and changes in total hSLEDAl and BILAG scores. Adverse events and laboratory values were assessed.
\end{abstract}

Results At week 52, $12.0 \%(3 / 25) \mathrm{RCI} / \mathrm{RCl}$ patients and $36.4 \%$ (4/11) placebo/RCI patients were responders using the novel index. The revised novel responder index demonstrated response rates of $48.0 \%(12 / 25)$ and $54.5 \%$ $(6 / 11)$ in the $\mathrm{RCl} / \mathrm{RCl}$ and placebo/RCl groups, respectively. Proportions of SRI responders were $40.0 \%$ (10/25) and $54.5 \%(6 / 11)$. In the RCl/RCl group, total hSLEDAl and BILAG scores declined from 10.0 and 15.7 at week 0 to 3.5 and 4.6 at week 52 , respectively. Reductions in the placebo/RCI group on switching were observed (mean hSLEDAl: 9.1-3.3; BILAG: 13.5-2.6). Other disease activity endpoints also improved in both groups. No new safety signals were observed during the OLE.

Conclusions $\mathrm{RCl}$ demonstrated durable effectiveness in patients with persistently active SLE despite moderatedose corticosteroid therapy. Switching from placebo resulted in reduced disease activity during the OLE. These data provide the foundation for evaluation of $\mathrm{RCl}$ in a robustly powered study.

\section{INTRODUCTION}

SLE is a systemic autoimmune disease that results in significant and irreversible damage to multiple organs. ${ }^{1}$ It is characterised by the deposition of immune complexes, and

\section{Key messages}

This post hoc analysis of results from a two-part pilot study consisting of an 8-week, prospective, randomised, double-blind, placebo-controlled phase, followed by a 44-week open-label extension (OLE), demonstrated sustained improvements in signs and symptoms of SLE in patients treated with repository corticotropin injection (RCl; H.P. Acthar Gel) for a total of 52 weeks.

- Patients who switched from placebo to $\mathrm{RCl}$ at the beginning of the OLE experienced improvements in signs and symptoms, which were comparable with those for patients who had received $\mathrm{RCl}$ in the first 8 weeks of the study.

- No new or unexpected safety or tolerability signals were observed during the OLE when compared with the double-blind period.

inflammation and organ damage are exacerbated by infiltration of leucocytes and the release of proinflammatory chemokines and cytokines. ${ }^{1-3}$ The aetiology of lupus is multifactorial, but B lymphocyte hyperactivity and loss of tolerance are major drivers of the disease. ${ }^{1}$ Affecting about $14-68$ in every 100000 persons in the USA, ${ }^{2}$ with similar prevalences in other parts of the world, ${ }^{45} \mathrm{SLE}$ is associated with significant morbidity and mortality. ${ }^{6}$

Patients with SLE may be treated with non-steroidal anti-inflammatory drugs, corticosteroids, hydroxychloroquine, methotrexate, azathioprine, ciclosporin, mycophenolate mofetil, tacrolimus, leflunomide or B lymphocyte-directed monoclonal antibodies (eg, rituximab and belimumab). ${ }^{5} 7$ Despite these options, there are important unmet treatment needs in SLE: conventional immunosuppressive agents have significant 
toxicities, and biological agents in randomised controlled trials have had modest effects, at best. ${ }^{8}$

Adrenocorticotropic hormone (ACTH) has been used since the 1950s for the treatment of patients with SLE. ${ }^{9}$ ACTH stimulates cortisol production by the adrenal gland $^{10}$ and also targets melanocortin receptors that are present on immune cells. ${ }^{10} 11$ Additionally, ACTH may increase levels of the anti-inflammatory cytokine interleukin (IL)-10 and decrease B lymphocyte proliferation and differentiation, as reflected by significant reductions in splenic B lymphocyte follicular and germinal centre cells and decreased levels of anti-double-stranded DNA (dsDNA) autoantibodies in a rodent model of lupus. ${ }^{11} 12$ Additional data suggest that RCI, but not placebo, attenuated IL-4/CD40 ligand-induced proliferation and immunoglobulin production in B lymphocytes isolated from healthy human volunteers. ${ }^{13}$

Repository corticotropin injection (RCI; H.P. Acthar Gel, Mallinckrodt ARD Inc., Bedminster, New Jersey, USA) is a prolonged-release formulation containing a highly purified porcine ACTH analogue. The US Food and Drug Administration has approved RCI for a number of indications, including for use during SLE exacerbations or as maintenance therapy in selected patients with SLE. ${ }^{14}$ Results were recently reported for the doubleblind and open-label extension (OLE) of a pilot study in patients with persistently active SLE, including rash and/ or arthritis, despite treatment with moderate-dose corticosteroids (NCT01753401). ${ }^{15} 16$ Although the primary endpoint of the double-blind portion of the study was not met, there were improvements in several other measures of disease activity for RCI versus placebo, including total hybrid Systemic Lupus Erythematosus Disease Activity Index (hSLEDAI), total British Isles Lupus Assessment Group (BILAG), Cutaneous Lupus Erythematosus Disease Area and Severity Index (CLASI) activity scores and tender and swollen joint count. ${ }^{16}$ This report summarises post hoc analyses that evaluated the efficacy of RCI over the entire 52-week study.

\section{METHODS}

\section{Study design}

This phase 4 pilot study (NCT01753401) consisted of an 8-week, randomised, double-blind, placebocontrolled treatment period followed by a 44-week OLE (figure 1). ${ }^{16}$ The first period explored the effect of RCI on disease activity in patients with SLE and persistently active arthritis and/or rash despite standard of care that included chronic/stable prednisone use for a minimum of 4 weeks before screening. The study was conducted at 20 sites in the USA. All patients provided written informed consent. All principal investigators, subinvestigators and study coordinators at each site were required to complete scale assessment training prior to enrolling any patients. Because this was a pilot study, serial assessments done by a single examiner were not required.

\section{Patients}

Inclusion and exclusion criteria for the double-blind period have been described. ${ }^{16}$ Briefly, adult patients (age $\geq 18$ years) were eligible if they met at least four

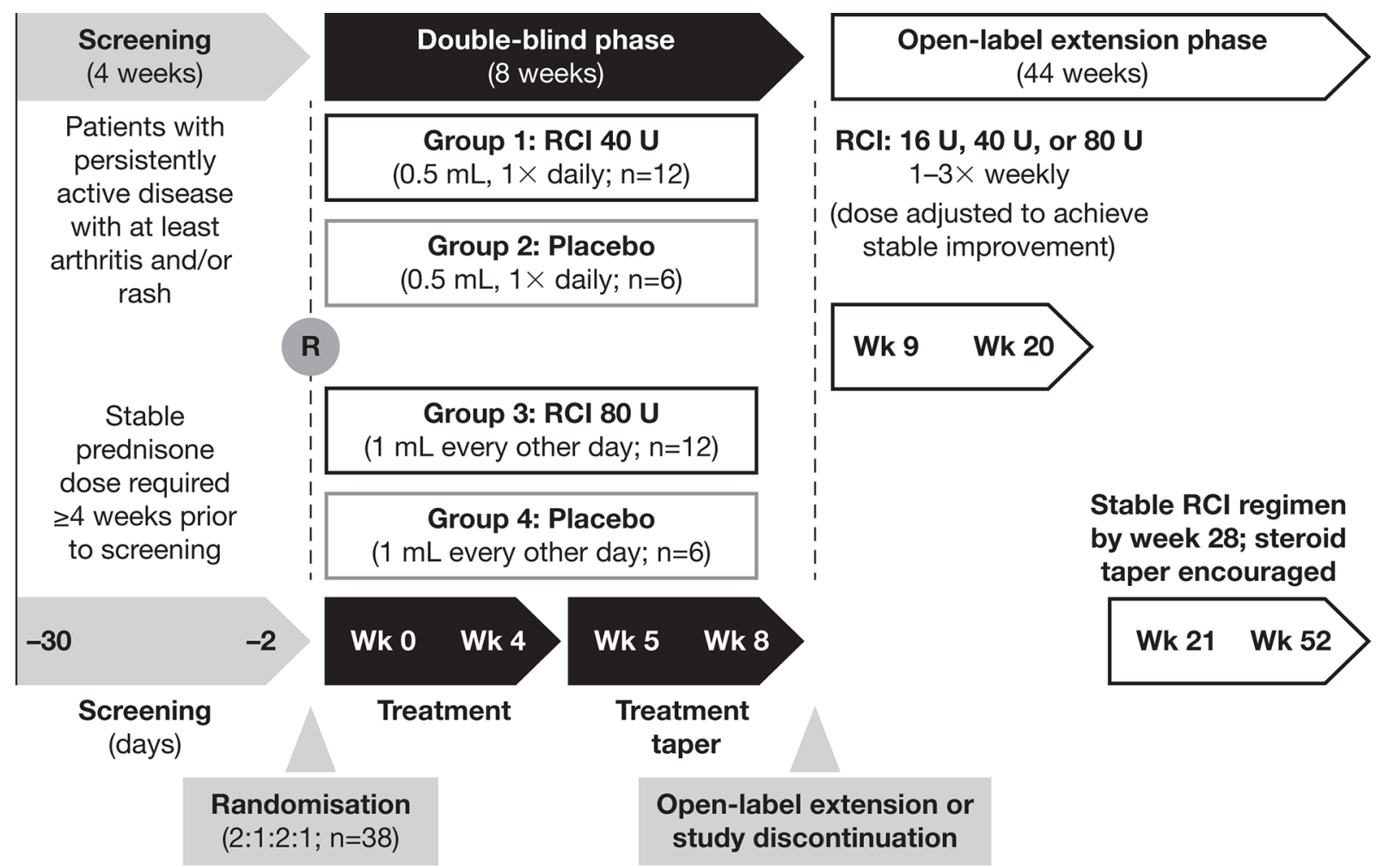

Figure 1 Study design. $\mathrm{R}$, randomisation; $\mathrm{RCl}$, repository corticotropin injection; Wk, week. 
of the American College of Rheumatology-revised diagnostic criteria for $\mathrm{SLE}^{17}$ and had active disease with joint and/or skin involvement sufficient to score $\geq 2$ on the hSLEDAI. Patients were also required to have a BILAG $^{18}$ score of A or B in the mucocutaneous and/ or musculoskeletal domains. All patients were required to be seropositive for, or have a documented history of, antinuclear antibodies and any one of the following: anti-dsDNA, anti-Smith or anticardiolipin antibodies. Persistent disease activity had to be evident despite the use of stable, moderate-dose corticosteroids (prednisone $7.5-30 \mathrm{mg}$ /day or equivalent) for at least 4 weeks prior to screening. All patients completing the doubleblind period were eligible to enter the OLE phase.

\section{Interventions}

In the double-blind phase, patients were assigned randomly to receive RCI $40 \mathrm{U}$ daily or $80 \mathrm{U}$ every other day by subcutaneous injection or to one of two volumematched placebo groups. During weeks 5-8 of the double-blind phase, RCI doses were tapered by reducing the frequency of administration to twice weekly according to a protocol-defined schedule. The initial RCI dose in the OLE was based on the study drug regimen at the completion of the double-blind period at week 8 . Patients receiving $0.2 \mathrm{~mL}$ (RCI $16 \mathrm{U}$ ), $0.5 \mathrm{~mL}$ (RCI $40 \mathrm{U}$ ) or $1.0 \mathrm{~mL}$ (RCI $80 \mathrm{U}$ ) injections of RCI or placebo at week 8 received RCI $16 \mathrm{U}, 40 \mathrm{U}$ or $80 \mathrm{U}$ twice weekly at the start of the OLE. Investigators were permitted to adjust the RCI regimen for safety or efficacy reasons from weeks 9-20 to achieve a stable RCI regimen (defined as consistent volume and frequency over at least 8 weeks) no later than week 28. Once a stable RCI regimen was achieved, steroid taper was encouraged but left to the investigator's judgement. The goal was to taper completely off steroids, but if this was not possible, attempts were made to taper to a low $(<7.5 \mathrm{mg}$ /day) daily dose.

\section{Assessments}

Efficacy variables included the hSLEDAI, ${ }^{19}$ BILAG2004, Physician's Global Assessment (PGA), ${ }^{20}$ Safety of Estrogens in Lupus Erythematosus National Assessment (SELENA) Flare Index (SFI), ${ }^{20-22}$ CLASI activity score ${ }^{23}$ and tender and swollen joint count. The hSLEDAI is identical to the SELENA-SLEDAI but uses the SLEDAI-2K definition for proteinuria and was used because it is able to capture ongoing proteinuria. Efficacy assessments were performed every 4 weeks except for hSLEDAI and SFI, which were assessed every 2 weeks for the first 8 weeks, then every 4 weeks for the remainder of the study. Safety was assessed by monitoring adverse events (AEs), physical examinations, measurements of vital signs and clinical laboratory investigations.

\section{Outcome measures}

A novel composite responder index, which was evaluated at 4-week intervals, defined a responder as a patient with a decrease in hSLEDAI score from 4 to 0 for arthritis and no worsening in other organ systems based on BILAG score, or a patient with a decrease in hSLEDAI score from 2 to 0 for rash and no worsening in other organ systems based on BILAG score. Worsening was defined as an increase in any BILAG score to a higher level (eg, a minor change in musculoskeletal or mucocutaneous systems could increase a D score to $\mathrm{C}$, which was considered worsening). Because of the stringency of the definition of 'worsening', a revision to the novel composite endpoint was made. The revised novel composite responder index redefined a responder as a patient with a decrease in hSLEDAI score from 4 to 0 for arthritis or from 2 to 0 for rash and no new BILAG A score and no more than one new BILAG B organ domain score. Additional assessments included the proportions of patients meeting the SRI, hSLEDAI decrease $\geq 4$ and rate of severe SFI flares at weeks 8 and 52. Other disease activity outcomes included changes in hSLEDAI, total BILAG score, PGA, changes in tender and swollen joint count for patients with score $>0$ at double-blind baseline and CLASI activity score for patients with score $>0$ at double-blind baseline. The proportion of patients achieving a reduction in daily prednisone dose to $<7.5 \mathrm{mg} /$ day by week 52 was also determined. Safety endpoints included AEs, serious AEs (SAEs) and SAEs and AEs leading to treatment discontinuation over the 52-week period.

\section{Statistical analyses}

All results reported used the modified intent-to-treat (mITT) population, defined as all randomised patients who had received at least one dose of study medication and who had any post-baseline efficacy or safety data. Descriptive statistics were used for continuous and categorical data. Quantitative endpoints were summarised using means and SD at each time point, and categorical endpoints were summarised using frequency counts and percentages at each time point. Non-responder imputation was used for missing data in the responder analyses. Analyses were performed using SAS V.9.3 software (Cary, North Carolina, USA).

\section{RESULTS}

\section{Patients}

A total of 38 patients were randomised to RCI $(n=26)$ or placebo $(\mathrm{n}=12)$ for the double-blind period; 33 completed visits through week 8 and entered the OLE (RCI/RCI: $\mathrm{n}=22$, placebo/RCI: $\mathrm{n}=11$ ), and 20 completed visits through week 52 (RCI/RCI: n=13, placebo/RCI: $\mathrm{n}=7$; figure 2). During the 52-week treatment period, 13 patients in the RCI/RCI group and 5 patients in the placebo/RCI discontinued treatment. Two patients (one in each group) withdrew consent and were not included in the mITT population. The mITT population consisted of 36 patients.

Demographic and disease characteristics at doubleblind baseline have been presented previously. ${ }^{16}$ Twelve patients $(33.3 \%)$ reported the use of immunosuppressant 


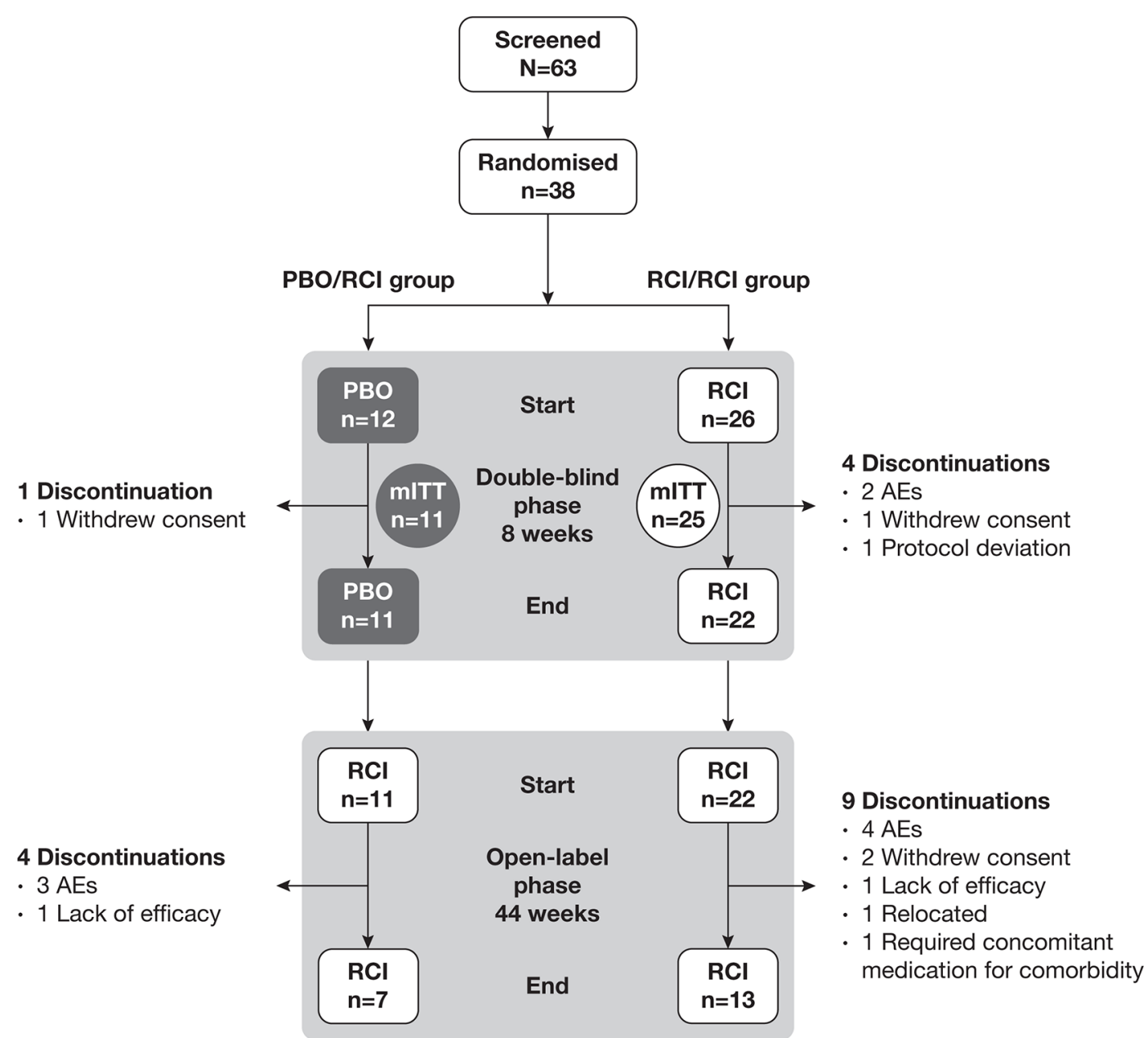

Figure 2 Patient disposition. AE, adverse event; mITT, modified intent-to-treat; PBO, placebo; RCI, repository corticotropin injection.

medications (azathioprine, methotrexate or mycophenolate mofetil) during the 52-week study period and $26(72.2 \%)$ used antimalarials. The mean prednisone daily dose was $10.0 \mathrm{mg}$ /day in the RCI/RCI group and $16.4 \mathrm{mg} /$ day in the placebo/RCI group. All measures of disease activity were higher for the placebo/RCI group versus the RCI/RCI group at the OLE baseline (table 1).

\section{Efficacy}

The proportions of patients defined as responders in the RCI/RCI group using the novel index at weeks 8 and 52 were $44.0 \%(11 / 25)$ and $12.0 \%(3 / 25)$, respectively. Applying the revised novel composite index (which reduced the unfavourable impact of the stringent BILAG worsening criteria by introducing the SRI definition for worsening in BILAG-2004), the proportions of responders were $60 \%(15 / 25)$ and $48.0 \%(12 / 25)$ at weeks 8 and 52, respectively (table 1 ). Improvements were also seen with standard disease activity indices (hSLEDAI decrease $\geq 4$ and SRI) at weeks 8 and 52 for the RCI/RCI group. Total hSLEDAI, BILAG-2004, CLASI activity and PGA scores and, to a lesser extent, tender and swollen joint count, continued to improve from weeks $8-52$ in these patients (figure 3 ). The proportion of patients that decreased their daily prednisone dose to $<7.5 \mathrm{mg}$ was $36.0 \%(9 / 25)$ at week 52 for the RCI/RCI group (table 1).

For patients who had received placebo during the double-blind period and who switched to RCI for the OLE, disease activity measures improved to levels comparable with those seen in patients in the RCI/RCI group (table 1). The effects of RCI were generally observed on or before 12-16 weeks after the switch from placebo to RCI (figure 3). The proportions of responders at weeks 8 and 52 were $27.3 \%(3 / 11)$ and $36.4 \%(4 / 11)$, respectively, using the novel index and $36.4 \%(4 / 11)$ and $54.5 \%(6 / 11)$ using the revised responder index, respectively (table 1 ). The proportion of patients that decreased their daily prednisone dose to $<7.5 \mathrm{mg}$ was $27.3 \%(3 / 11)$ at week 52 for the placebo/RCI group (table 1$)$.

Of the 12 patients receiving immunosuppressive therapies at the start of the trial, two discontinued concomitant immunosuppressants (mycophenolate mofetil, azathioprine; one patient each) and one reduced their dose of methotrexate from $12.5 \mathrm{mg}$ daily to $7.5 \mathrm{mg}$ during the OLE period. 
Table 1 Efficacy endpoints at weeks 8 and 52 (mlTT population)

\begin{tabular}{|c|c|c|c|c|c|c|}
\hline & \multicolumn{2}{|c|}{ Week 0 (baseline) } & \multicolumn{2}{|c|}{ Week 8 (OLE baseline) } & \multicolumn{2}{|l|}{ Week 52} \\
\hline & $\mathrm{RCl} / \mathrm{RCl}$ & Placebo/RCI & $\mathrm{RCl} / \mathrm{RCI}$ & Placebo/RCI & $\mathrm{RCl} / \mathrm{RCI}$ & Placebo/RCI \\
\hline Total hSLEDAI & $10.0(3.3)$ & $9.8(2.1)$ & $5.8(3.0)$ & $9.1(3.4)$ & $3.5(3.5)$ & $3.3(2.5)$ \\
\hline CLASI activity score* & $6.7(6.3)$ & $7.4(6.6)$ & $3.9(4.3)$ & $7.0(7.0)$ & $1.3(1.6)$ & $0.5(0.8)$ \\
\hline Tender and swollen joint count* & $7.4(5.8)$ & $5.1(4.9)$ & $1.4(2.3)$ & $2.2(3.2)$ & $0.9(2.5)$ & $1.6(2.6)$ \\
\hline Novel responder index, n/N (\%) & $\mathrm{n} / \mathrm{a}$ & $\mathrm{n} / \mathrm{a}$ & $11 / 25(44.0)$ & $3 / 11(27.3)$ & $3 / 25(12.0)$ & $4 / 11(36.4)$ \\
\hline Revised novel responder index, † n/N (\%) & $\mathrm{n} / \mathrm{a}$ & $\mathrm{n} / \mathrm{a}$ & $15 / 25(60.0)$ & $4 / 11(36.4)$ & $12 / 25(48.0)$ & $6 / 11(54.5)$ \\
\hline SLE responder index, n/N (\%) & $\mathrm{n} / \mathrm{a}$ & $\mathrm{n} / \mathrm{a}$ & $13 / 25(52.0)$ & $1 / 11(9.1)$ & $10 / 25(40.0)$ & $6 / 11(54.5)$ \\
\hline hSLEDAl decrease $\geq 4, n / N(\%)$ & $\mathrm{n} / \mathrm{a}$ & $\mathrm{n} / \mathrm{a}$ & $15 / 25(60.0)$ & $1 / 11(9.1)$ & $10 / 25(40.0)$ & $6 / 11(54.5)$ \\
\hline Prednisone $<7.5 \mathrm{mg} /$ day, $\mathrm{n} / \mathrm{N}(\%)$ & $\mathrm{n} / \mathrm{a}$ & $\mathrm{n} / \mathrm{a}$ & $\mathrm{n} / \mathrm{a}$ & $\mathrm{n} / \mathrm{a}$ & 9/25 (36.0) & $3 / 11(27.3)$ \\
\hline
\end{tabular}

Data reported as mean (SD) unless indicated otherwise.

${ }^{*}$ Calculated for patients with score $>0$ at double-blind baseline.

†Novel responder index calculated using SRI definition for BILAG worsening (no new BILAG A and not more than one new BILAG B).

BILAG, British Isles Lupus Assessment Group; CLASI, Cutaneous Lupus Erythematosus Disease Area and Severity Index; hSLEDAI, hybrid Systemic Lupus Erythematosus Disease Activity Index; mITT, modified intent-to-treat; n/a, not applicable; OLE, open-label extension; RCI, repository corticotropin injection; SFI, Safety of Estrogens in Lupus Erythematosus National Assessment (SELENA) Flare Index.

\section{Safety and tolerability}

The incidences of treatment-emergent AEs (TEAEs) and treatment-related AEs in the placebo/RCI and $\mathrm{RCI} / \mathrm{RCI}$ groups were similar over the 52-week study period (table 2). One death attributed to Klebsiella sepsis, and multiorgan failure was reported in the RCI/RCI group. This event was previously reported. ${ }^{16}$ Three other RCI/RCI patients experienced serious TEAEs (one patient with gastro-oesophageal reflux and chest discomfort (double-blind phase), one with haemorrhagic ovarian cyst and viral infection (double-blind phase) and pelvic infection and lower abdominal pain (OLE) and one with pelvic abscess (OLE)). Of the four patients experiencing serious TEAEs, two were classified as probably or definitely related to the study drug (chest discomfort/gastro-oesophageal reflux and pelvic abscess). Four placebo/RCI patients experienced serious TEAEs (viral infection, non-cardiac chest pain, pyelonephritis and SLE flare with hospitalisation). All of these events occurred during the OLE, and only one (pyelonephritis) was classified as possibly related to study drug.

TEAEs leading to study discontinuation occurred in six patients in the RCI/RCI group (three during the doubleblind phase and three during the OLE) and included one severe event, a false-positive hepatitis C screening test result (after withdrawal from the study, this patient died of Klebsiella sepsis). Of the three TEAEs leading to discontinuation in the placebo/RCI group, one occurred during the double-blind phase and two during the OLE. Only one (ulcerative keratitis during the OLE) was classified as severe and was not related to study drug. There were no clinically significant changes in physical examination findings or vital signs, including blood pressure or clinical laboratory tests, during the 52-week treatment period.

\section{DISCUSSION}

The results of the post hoc analyses for this two-part, 52-week, phase 4 study demonstrated that the addition of RCI to standard of care led to relatively rapid and sustained improvements in a patient population with SLE and refractory arthritis and/or rash despite moderate-dose corticosteroid therapy.

Patients who received RCI throughout the 52-week treatment period had a durable response to therapy, while patients who crossed over from placebo to RCI at the end of the double-blind period at week 8 experienced improvements in multiple measures of disease activity during the OLE. The degree of improvement was generally comparable with that observed with RCI treatment during the double-blind phase and was achieved by about 12-16 weeks after RCI was initiated.

The effects of RCI during the 52-week treatment period were evaluated in post hoc analyses using a number of validated outcome measures, including the hSLEDAI, BILAG-2004, CLASI, PGA, SRI, assessments of tender and swollen joints, as well as two novel composite responder indices. Although the primary endpoint of response using the novel index was not met for the double-blind phase, ${ }^{16} \mathrm{RCI}$ demonstrated consistent improvement in results across multiple outcome measures, such as the SLEDAI and BILAG over 52 weeks. Consistency of results across multiple measures was seen in the tender and swollen joint count and CLASI scores among patients with counts and scores $>0$ 

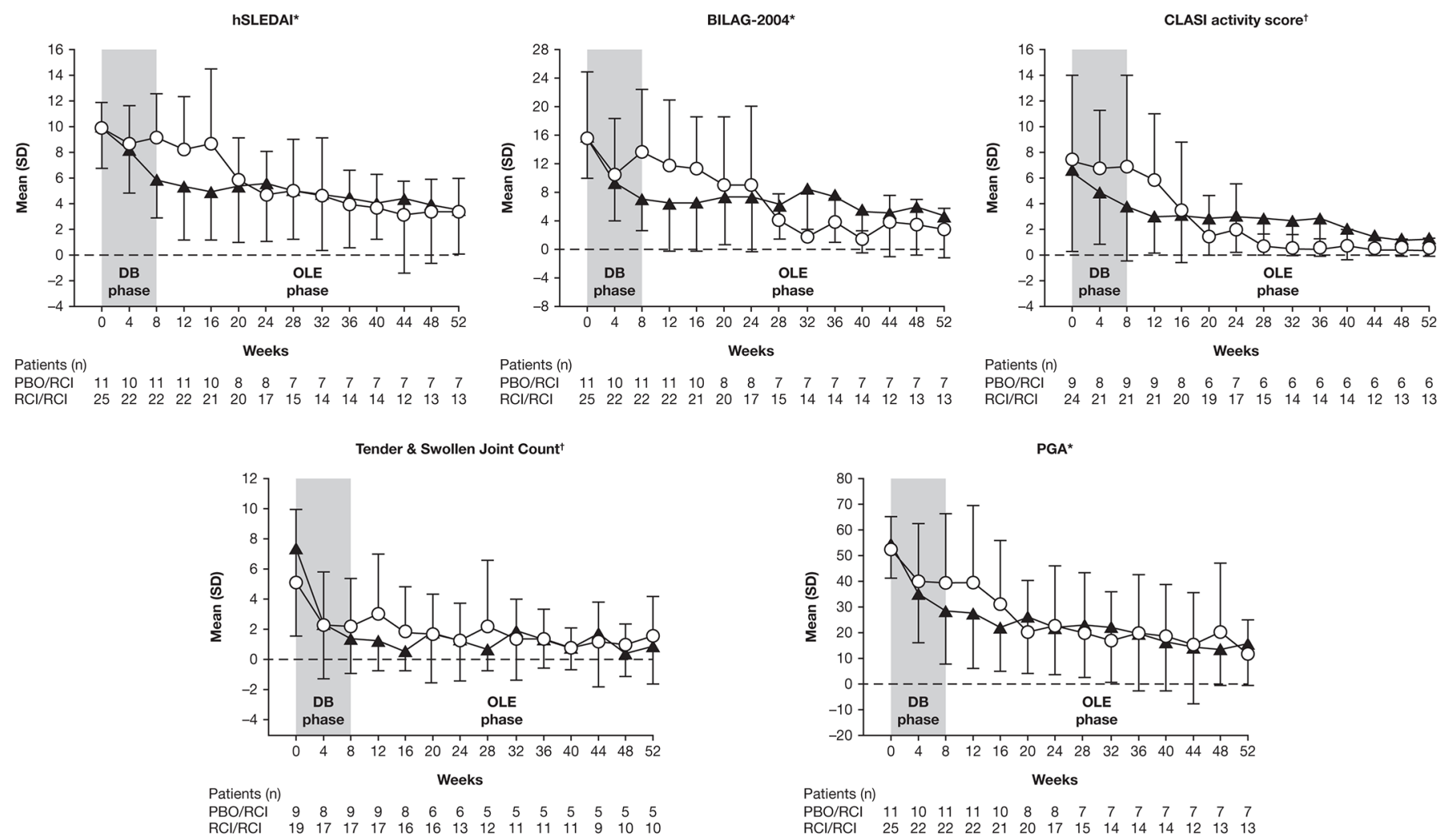

Figure 3 Disease activity measures over time. *Mean and SD were calculated on the basis of the observed data at each time point. †Mean and SD were calculated using only scores $>0$ at week 0. BILAG, British Isles Lupus Assessment Group; CLASI, Cutaneous Lupus Erythematosus Disease Area and Severity Index; DB, double-blind; hSLEDAI, hybrid Systemic Lupus Erythematosus Disease Activity Index; OLE, open-label extension; PBO, placebo; PGA, Physician's Global Assessment; RCl, repository corticotropin injection.

at baseline. Improvements in the novel organ-specific responder index were similar to those for the revised novel responder index at week 8 , but the response rate using the novel organ-specific responder index was considerably lower at week 52 than the rate using the revised index. Inconsistency between the week 52 responder index rates as opposed to the rates at week
8 most likely relates to the longer exposure and thus greater opportunity for mild flares to be captured due to the more stringent BILAG threshold of worsening that was incorporated into the response definition of the novel responder index (no worsening in other organ systems on the basis of BILAG score) versus the revised novel responder index (no new BILAG

Table 2 Summary of TEAEs and treatment-related AEs over 52 weeks*

\begin{tabular}{|c|c|c|}
\hline Parameter & Placebo/RCI (n=11) & $\mathrm{RCl} / \mathrm{RCl}(\mathrm{n}=25)$ \\
\hline Any TEAE & $11(100)$ & $23(92.0)$ \\
\hline Any severe TEAE† & $3(27.3)$ & $2(8.0)$ \\
\hline Any treatment-related $\mathrm{AE} \neq$ & $6(54.5)$ & $14(56.0)$ \\
\hline Any TEAE leading to study discontinuation & $3(27.3)$ & $6(24.0)$ \\
\hline Any serious TEAE & $4(36.4)$ & $4(16.0)$ \\
\hline Any TEAE resulting in death & 0 & $1(4.0)$ \\
\hline
\end{tabular}

Data reported as $\mathrm{n}(\%)$.

${ }^{*}$ AEs classified into System Organ Class and preferred terms using the Medical Dictionary for Regulatory Activities, V.15.1.

†AEs were considered severe if the severity of an event was missing.

$\ddagger A E s$ were considered treatment related if the relationship to study medication was possibly related, probably related, definitely related or missing.

$\mathrm{AE}$, adverse event; $\mathrm{RCl}$, repository corticotropin injection; TEAE, treatment-emergent adverse event. 
A score and no more than one new BILAG B organ domain score). BILAG worsening of any degree is a particularly stringent endpoint as minor variations in BILAG disease activity or laboratory variables can too easily trigger worsening domain scores. ${ }^{24}$ The revision defined BILAG worsening based on the original SRI. While stringent analyses are important to demonstrate substantial improvement, they can also be challenging to use to demonstrate treatment effects in small studies. Therefore, this revision to the novel responder index may prove useful in future pilot studies of treatment when looking for indication of a potential response. Taken together, the efficacy results provide preliminary, randomised, controlled evidence that RCI treatment results in reductions in disease activity and corticosteroid requirements in patients with SLE who have persistent disease despite moderate-dose corticosteroid therapy.

RCI was well tolerated in the pilot study. No new safety signals were observed during the 52-week treatment period. Important study limitations include the small sample and the lack of inferential statistical analyses, meaning that conclusions regarding clinical improvement with long-term RCI must be viewed with caution.

In conclusion, the results of this pilot 52-week study support RCI as a treatment option for patients with SLE disease manifestations that are unresponsive to moderate-dose corticosteroid therapy. Preclinical evidence in healthy humans demonstrating direct, steroidindependent effects of RCI on the function of activated human B lymphocytes supports a potential unique mechanism of action for this agent in the treatment of autoimmune diseases characterised by aberrant humoural immune responses. ${ }^{13}$ Additional studies to better understand how differential in vitro signaling in response to RCI might contribute to its clinical efficacy are ongoing. Further evaluation of RCI in a well-powered clinical trial in SLE is underway (NCT02953821).

Acknowledgements The authors would like to thank Shanique Smythe and Maya Das (formerly of Mallinckrodt) for contributions to study monitoring and data collection. The first draft of the manuscript was prepared by academic and industry authors, with professional medical writing and editorial assistance provided by Robert Rhoades, Caudex Health, Oxford, UK, and funded by Mallinckrodt.

Contributors RAF and PMB were involved in the design of the study, interpretation and analysis of data, and drafting of the manuscript or revising it for critically important intellectual content. MM and EZ were involved in the interpretation and analysis of data and drafting of the manuscript or revising it for critically important intellectual content. All authors provided final approval of the submitted manuscript and vouch for the completeness and accuracy of the data and data analyses and for the fidelity of the study to the protocol.

Funding Mallinckrodt Pharmaceuticals sponsored this study and provided the study drug, designed the study, conducted the study, collected the data, monitored the conduct of the study and performed statistical analyses in collaboration with the contract research organisation (INC Research, Raleigh, North Carolina, USA) and the external principal and site investigators. The sponsor also provided funding for professional medical writing and editorial assistance.

Competing interests RAF has been a paid consultant to Mallinckrodt Pharmaceuticals as well as the study and a site principal investigator. MM is a paid consultant to Mallinckrodt Pharmaceuticals. EZ is an employee of Mallinckrodt
Pharmaceuticals. PMB is an employee of Mallinckrodt Pharmaceuticals and holds stock or stock options in the company.

Patient consent Obtained.

Ethics approval The protocol was approved by the Institutional Review Boards at all participating centres, and the study was conducted according to the principles of the Declaration of Helsinki and Good Clinical Practice.

Provenance and peer review Not commissioned; externally peer reviewed. Data sharing statement № additional data are available.

Open Access This is an Open Access article distributed in accordance with the terms of the Creative Commons Attribution (CC BY 4.0) license, which permits others to distribute, remix, adapt and build upon this work, for commercial use, provided the original work is properly cited. See: http://creativecommons.org/ licenses/by/4.0/

(c) Article author(s) (or their employer(s) unless otherwise stated in the text of the article) 2017. All rights reserved. No commercial use is permitted unless otherwise expressly granted.

\section{REFERENCES}

1. Gottschalk TA, Tsantikos E, Hibbs ML. Pathogenic inflammation and its therapeutic targeting in systemic lupus erythematosus. Front Immunol 2015;6:550.

2. Bailey T, Rowley K, Bernknopf A. A review of systemic lupus erythematosus and current treatment options. Formulary 2011;46:178-94.

3. Liao X, Pirapakaran T, Luo XM. Chemokines and chemokine receptors in the development of lupus nephritis. Mediators Inflamm 2016;2016:1-15

4. Arnaud L, Fagot JP, Mathian A, et al. Prevalence and incidence of systemic lupus erythematosus in France: a 2010 nation-wide population-based study. Autoimmun Rev 2014;13:1082-9.

5 Gordon C, Amissah-Arthur MB, Gayed M, et al. British Society for Rheumatology Standards, Audit and Guidelines Working Group. The British Society for Rheumatology guideline for the management of systemic lupus erythematosus in adults. Rheumatology 2018;57:e1-e45.

6. Knight AM, Weiss PF, Morales $\mathrm{KH}$, et al. National trends in pediatric systemic lupus erythematosus hospitalization in the United States: 2000-2009. J Rheumatol 2014;41:539-46.

7. Bertsias G, loannidis JP, Boletis J, et al. EULAR recommendations for the management of systemic lupus erythematosus. Report of a Task Force of the EULAR Standing Committee for International Clinical Studies Including Therapeutics. Ann Rheum Dis 2008;67:195-205.

8. Oon S, Wilson NJ, Wicks I. Targeted therapeutics in SLE: emerging strategies to modulate the interferon pathway. Clin Trans/ Immunology 2016;5:e79.

9. Harris-Jones JN. The role of ACTH and cortisone in the treatment of systemic lupus erythematosus. Postgrad Med J 1956;32:145-9.

10. Montero-Melendez T. ACTH: The forgotten therapy. Semin Immunol 2015;27:216-26.

11. Loram LC, Culp ME, Connolly-Strong EC, et al. Melanocortin peptides: potential targets in systemic lupus erythematosus. Inflammation 2015;38:260-71.

12. Decker DA, Grant C, Oh L, et al. Immunomodulatory effects of H.P. Acthar Gel on B cell development in the NZB/W F1 mouse model of systemic lupus erythematosus. Lupus 2014;23:802-12.

13. Olsen NJ, Decker DA, Higgins P, et al. Direct effects of HP Acthar Gel on human B lymphocyte activation in vitro. Arthritis Res Ther 2015;17:300

14. Mallinckrodt Pharmaceuticals. Acthar Gel (repository corticotropin injection). Prescribing information. www.acthar.com/pdf/Acthar-PI. pdf (accessed 22 May 2017).

15. Becker P, Furie R, Mitrane M, et al. Repository corticotropin injection $(\mathrm{RCl})$ attenuates disease activity in patients with persistently active systemic lupus erythematosus (SLE) requiring corticosteroids: results from a 44-week open-label extension study. Ann Rheum Dis 2016;75:297-8

16. Furie R, Mitrane M, Zhao E, et al. Efficacy and tolerability of repository corticotropin injection in patients with persistently active SLE: results of a phase 4, randomised, controlled pilot study. Lupus Sci Med 2016;3:e000180.

17. Hochberg MC. Updating the American College of Rheumatology revised criteria for the classification of systemic lupus erythematosus. Arthritis Rheum 1997;40:1725.

18. Isenberg DA, Rahman A, Allen E, et al. BILAG 2004. Development and initial validation of an updated version of the British Isles Lupus 
Assessment Group's disease activity index for patients with systemic lupus erythematosus. Rheumatology 2005;44:902-6.

19. Thanou A, Chakravarty E, James JA, et al. Which outcome measures in SLE clinical trials best reflect medical judgment? Lupus Sci Med 2014;1:e000005.

20. Petri M, Buyon J, Kim M. Classification and definition of major flares in SLE clinical trials. Lupus 1999;8:685-91.

21. Buyon JP, Petri MA, Kim MY, et al. The effect of combined estrogen and progesterone hormone replacement therapy on disease activity in systemic lupus erythematosus: a randomized trial. Ann Intern Med 2005;142:953-62.

22. Petri M, Kim MY, Kalunian KC, et al. Combined oral contraceptives in women with systemic lupus erythematosus. $N$ Engl $\mathrm{J} \mathrm{Med}$ 2005;353:2550-8.

23. Albrecht J, Taylor L, Berlin JA, et al. The CLASI (Cutaneous Lupus Erythematosus Disease Area and Severity Index): an outcome instrument for cutaneous lupus erythematosus. J Invest Dermatol 2005;125:889-94.

24. Furie RA, Petri MA, Wallace DJ, et al. Novel evidence-based systemic lupus erythematosus responder index. Arthritis Rheum 2009;61:1143-51. 